\title{
La race bovine rouge du sud de l'Anatolie (güney anadolu kirmizisi)
}

\author{
par R. FERRANDO*, R. FIASSON** et Feridun GORGULU***
}

\begin{abstract}
RÉSUMÉ
Les auteurs décrivent la race, originaire de Syrie et du Lıban où elle est dénommée : race Beledie de Damas ou d'Alep. Parfaitement adaptée à la chaleur et à la sécheresse, elle continue d̀ donner des rendements en lat élevés, sı elle est bien nourrie. La production laıtıère annuelle $(2416 \pm 84,2 \mathrm{l})$, la longueur de la période de lactation $(274,4 \pm 4,07 \mathrm{~J})$, le taux moyen butyreux $(3,515 \pm 0,02$ p. 100), la production selon les mois de l'année el l'effet de l'âge sur la lactation sont calculés à partir d'un matérıel d'étude portant sur 215 vaches de 52 familles.

Dans leurs conclusions, les auteurs indıquent qu'un effort de sélection coordonné et suivi permettrail d'amélıorer une race intéressante el d'obtenır des sujets aptes à l'exportation et dénoncent l'erreur qui consiste à ımporter des races très évoluées pour éviter d'accomplır un long travail de sélection, sacrifiant ainsi la réalité au mythe.
\end{abstract}

En 1941 I'un de nous (2) attiralt déà l'attention sur cette race originaire des régions riches de la Syrie et du Lıban (1). Elle est dénommée dans ces pays : Race Bélédie de Damas ou d'Alep (cf. fig. 1). On l'appelle encore en Turquie, dans la province de Balıkésir, l'«Egyptıenne». Elle présente, à notre avis, un intérêt certain. II est regrettable qu'elle soit abandonnée au profit de race comme la Frisonne nullement adaptée aux contrées dont il est ici question. Cette race fut, il y a une quinzaıne d'années, exportée en Tunisı et en Algérie. Elle pourrait peupler avantageusement de nombreuses régions circumméditerranéennes et même être exploitée dans d'autres pays du monde au clımat méditerranéen voire subtropical. Malheureusement ce qu'un de nous a pu qualıfier de snobisme zootechnique est un

\footnotetext{
* Ecole Natıonale vétérınaıre, 94-Alfort, France.

** Conseiller d'élevage auprès du Ministère de l'Agrıculture, Ankara.

*** Chef du Service du Herd-Book au Minıstère de l'Agriculture, Ankora.
}

défaut aussi répandu que difficile à combattre. Le sens des réalités doit pourtant être la première qualité des éleveurs et des services de l'élevage. II est comme le bon sens dont on sait ce que DESCARTES a dit à son sujet.

\section{MORPHOLOGIE}

Lo race est médiolıgne et eumétrique. Un examen approfond permet de noter un mélange de caractères qui font hésiter à la classer par$\mathrm{mI}$ les subconcaves. II est vraisemblable que des croisements multıples, voire même des apports de sang zébu, sont intervenus dans sa formation.

Ces régions de l'Asie où vit la race furent un lieu de passage : des crolsements se sont fatalement prodults. La vache Bélédie peut très bien avoır dans ses ancêtres des représentants de la race asıatıque des steppes (bos taurus asiaticus) qui est rectılıgne. En effet, on trouve porfois chez elle des cornes en coupe ou 


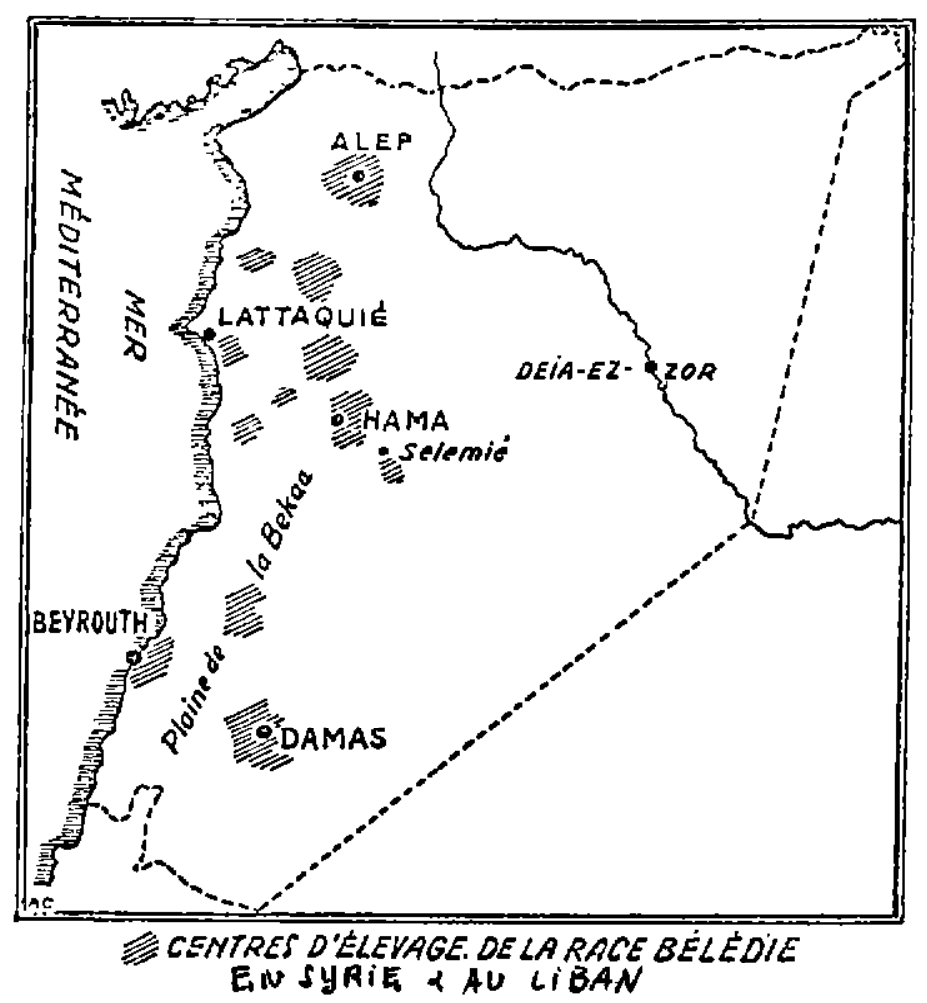

Fig. 1.

en lyre haute. Mais on trouve également, ef le plus souvent, des cornes en couronne ou en crochet, parfois même des cornes très atrophiées indiquant un ancêtre concave et même peutêtre un ancêtre sans cornes. Ajoutons à cela le chignon parfois saillant ef la queve en crosse et nous pouvons encore songer d̀ des ancêtres convexes. Le bos tourus Scythicus ne seralt-il pas allé jusqu'en Asie mineure? D'autre part, MM. GAILLARD et le docteur PORTET, dans «La Faune momifiée de l'ancienne Egypte», indiquent et prouvent qu'une race bovine sans cornes vécut autrefois sur les bords du Nil. Des migrations de peuples, des échanges multiples s'effectuèrent entre I'Egypte et la Syrie. Ce dernier pays fut toujours un carrefour où les peuples et les races anımales se sont sans arrêt affrontés ef mêlés. Pourquoi donc les animaux de l'espèce bovine n'en auralent-ıls pas fait autant? Certes ce sont là de simples hypothèses; cependant, en ce qui concerne la race Bélédie, l'étude de sa morphologie dévoile son origine métısse.

On est surtout frappé de la très grande finesse des sujets de cette race. La vache Bélédıe est un peu la gazelle de l'espèce. Le milieu, le climat, l'aptitude sont causes de cet affinement qui se rencontre même chez le taureau.

La tête est fine et courte, portant une dépression frontale légèrement accusée. Le profil est subconcave. Le mufle est moyen très légèrement retroussé.

Les cornes sont en général insérées sur la ligne du chignon. Le plus souvent elles sont ramenées en couronne ou en crochets. On trouve aussi des cornes en coupes ou en lyre haute et, fréquemment, des cornes atrophiées, petıtes, rabougries, comme rognées; parfols il n'y a qu'une corne. Cette grande variété de cornage nous a fait hésiter pour classer cette race selon la méthode de BARON.

L'encolure est svelte chez la vache, très musclée chez le taureau et le bouf. Le mâle a un garrot large et haut. Le train antérieur est développé.

Le fanon est abondant.

La poitrine est large, ample, profonde, bien descendue. 


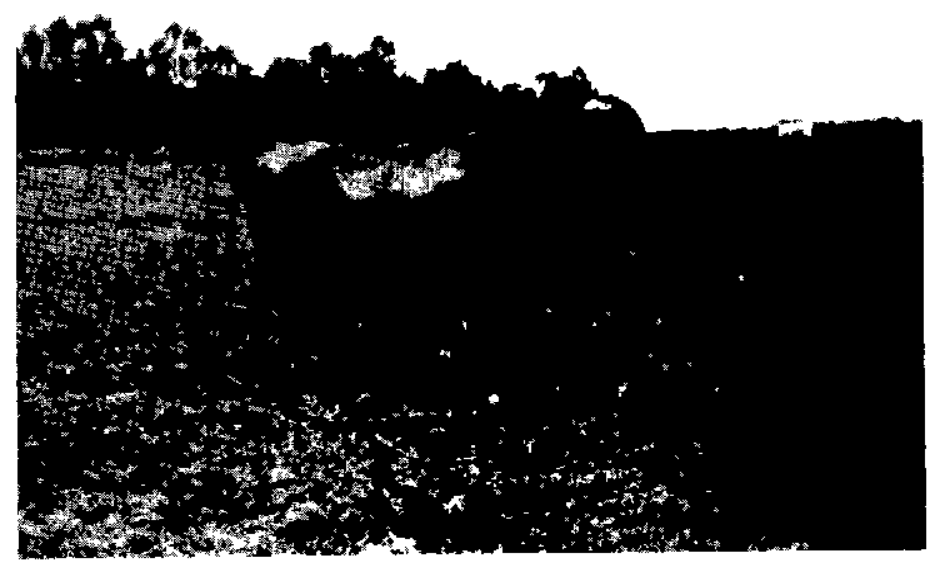

Taureau Rouge du Sud. Dépôt de bovins de BOZTEPE-ANTALYA.

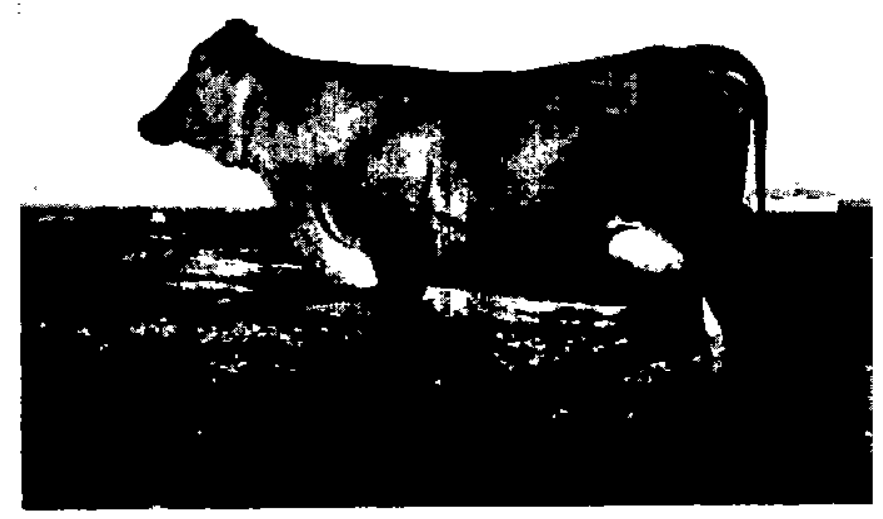

Vache Beledie. 
Le dessus est drolt. Les hanches sont assez saillantes et larges comme tout le bassin. Lo queue est longue et fine. Son attache, quelquefois en cimier, est le plus souvent horizontale. La fesse est drolte, la cuisse plate et maigre. Les membres solides, très fins, très nerveux, ont d'excellents aplombs.

La peau est mince, souple et mobile; les polls sont courts et brillants.

Les caractères laitiers sont très développés. La mamelle est volumineuse, bien conformée, très irriguée. On peut reprocher à certains sujets la petitesse de leurs trayons, ce qui n'enlève rien à leurs qualités laitıères.

Taille : 1 mètre 45 à 1 mètre 55 chez le mâle.

1 mètre 35 à 1 mètre 40 chez la femelle.

Poids : 600 à 700 kilos chez le mâle.

400 à 500 kilos chez la femelle

(cf. Tableau I pour les mensurations).

La robe est brune. Le mufle, le dessous de la langue, les paupières, le pourtour de l'anus ef de la vulve sont pigmentés. Chez le mâle il existe une cupule remontant assez haut. Les cornes, blanches à la base, ont les extrémités noires. SI ces caractères de pigmentation sont généraux et constants, la robe varie quelque peu du fauve clarr au brun foncé.

Dans le premier cas, la robe fauve clair ressemble à celle de la race Tarentase, avec des lunettes et une bordure de poils noirs autour du mufle. Les extrémités, les bracelets de la couronne et le toupillon de la queve sont noirs. Les parties postérieures des canons (ligne des tendons) sont recouvertes de polls gris argent qui forment transition entre la muqueuse du mufle et sa bordure noire. Le mâle est toujours plus foncé. II possède des lunettes plus accentuées. On trouve chez lui des charbonnures sur le cou, les épaules et le fanon. Les poils du fourreau sont noirs.

Dans un second cas, la robe, qui est fauve foncé tirant légèrement sur le brun fauve, possède les mêmes particularıtés que la précédente.

Enfin quelques individus possèdent une robe brune, presque noire.

On préfère la première robe.

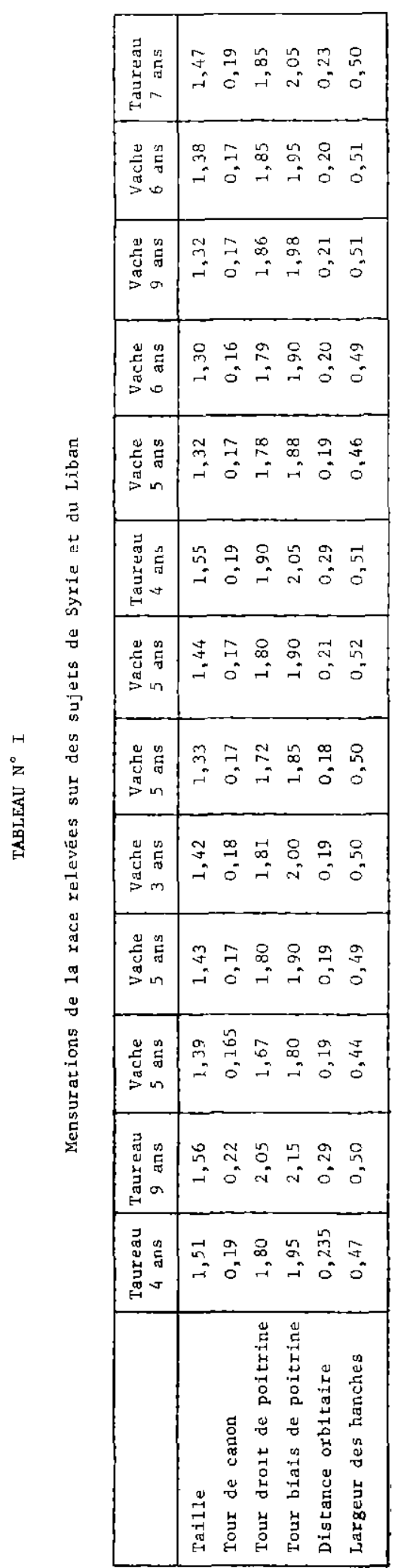




\section{APTITUDES}

La race, dont toute la conformation révèle la finesse, est bonne laitière ; on peut même dire excellente laitı̀re, si on consıdère le poids de la vache Bélédıe et le milieu où elle vit.

La production est de 3.000 à $3.500 \mathrm{~kg}$ de lait par lactation. En considérant la courte durée de cette dernière : 195 à 300 jours (245 jours en moyenne), on voit que la quantité journalıère est assez forte. Des vaches d'élite arrivent à produire, en Syrie et au Liban, durant les deux premıers mols de leur lactation, 15, 18 et même 23 kilogrammes de lait par jour. Ce lait est assez riche en matière grasse ; 11 en faut 22 d̀ 25 litres pour faire un $\mathrm{kg}$ de beurre.

Si la vache Bélédie est une excellente laıtı̀re, sa pérıode de lactation est relativement courte. On note, pendant la lactation, quelques chutes de débit dues à l'alımentatıon ırréguhère. Enfin, la sécrétion semble parfois se tarir en quelques semaines (fig. 2 et 3 ).

De caractère très doux, les taureaux et les boufs sont d'excellents animaux de travall. Leur train antérieur très développé, leurs membres solides permettent un effort régulier et soutenu.
Les qualités précédentes ef l'absence totale de précocité, font de ces sujets de très médiocres anımcux de boucherie (3). Leur rendement varie de 45 à 48 p. 100.

\section{LA RACE EN TURQUIE}

C'est de l'autre côté des monts du Taurus qui lımitent au Sud le plateau anatolien, où sont les planes d'Antalya, d'Adana et de Hatay (I'ancien sandiak d'Alexandrette) que se situent les régıons où s'élève la race bovine Rouge du Sud (Guney Anadolu Kirmizısi). On compte 550.000 têtes de cette race pour l'ensemble du territoire turc (cf. fig. 4).

Les sujets rencontrés icı sont de robe froment, parfois virant au cerise. Ils frappent toujours l'observateur par la longueur de leurs membres postérieurs, leur taille élevée et une bosse plus ou moins apparente selon les individus.

La race est ICı particulıèrement bıen adaptée au climat semitropical des plaines qui bordent la mer Méditerranée, comme elle l'est aussı aux vastes étendues situées sur la frontière de Syrie et d'Irak : province de Gazıantep, Maras, Urfa,

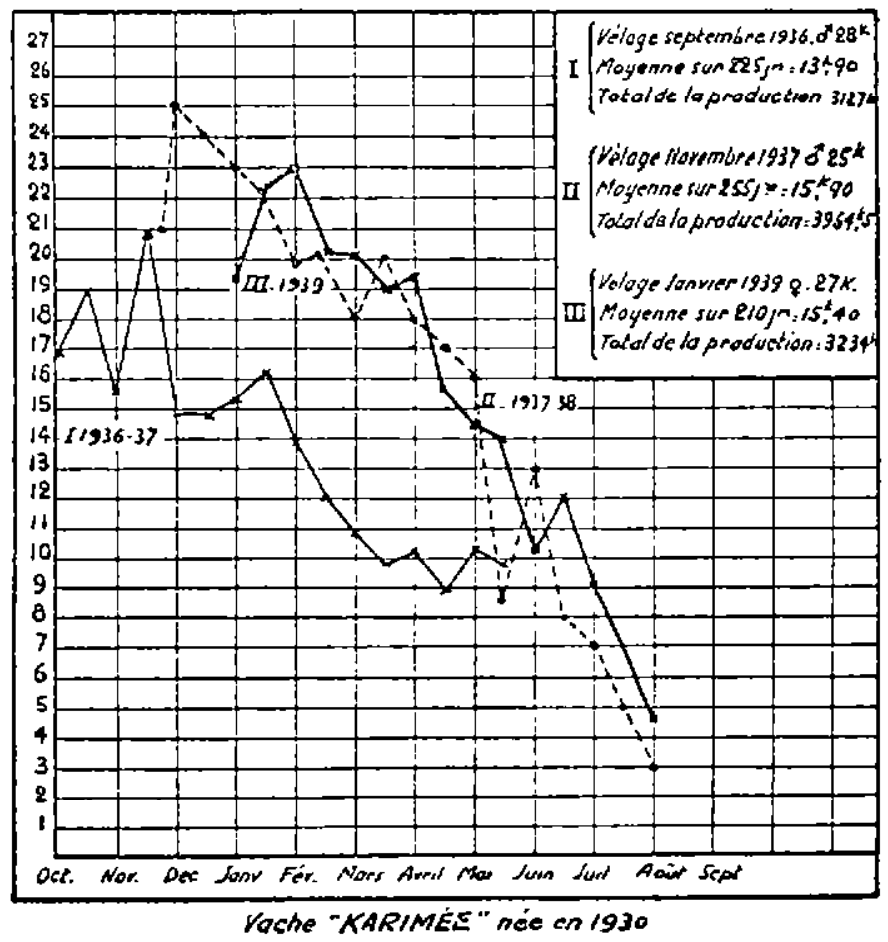

Fig. 2. 


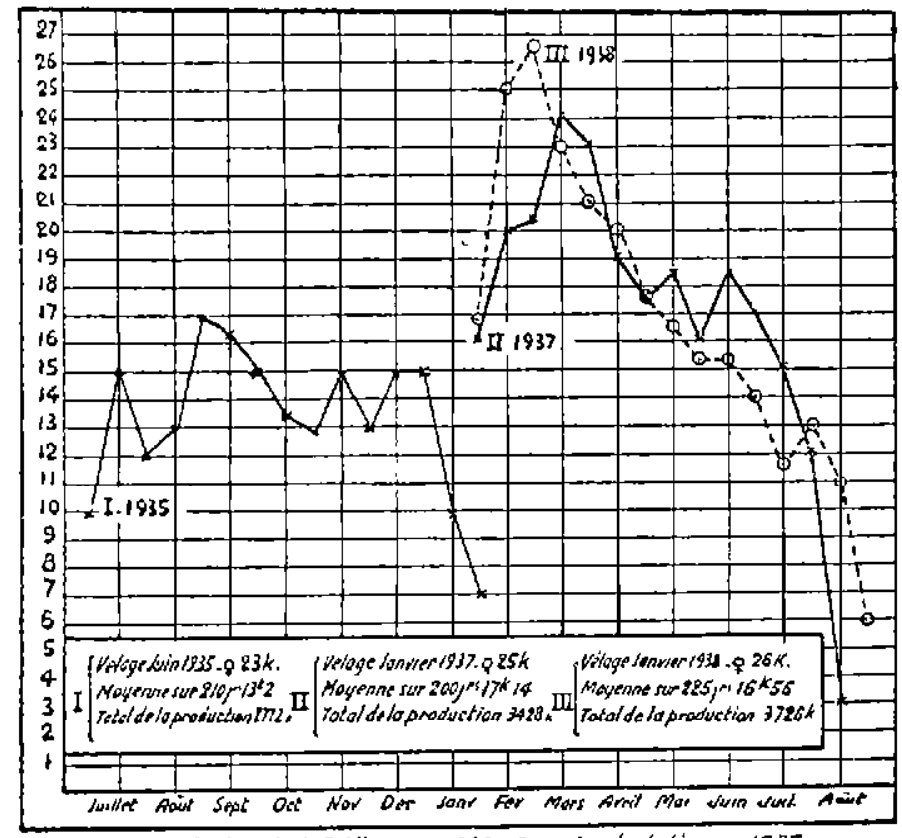

Voche "AMIRA" Wee en 1932. Premino loclation en 1935 an 1936 so production est de 3.029kalogi de lat.

Fig. 3.

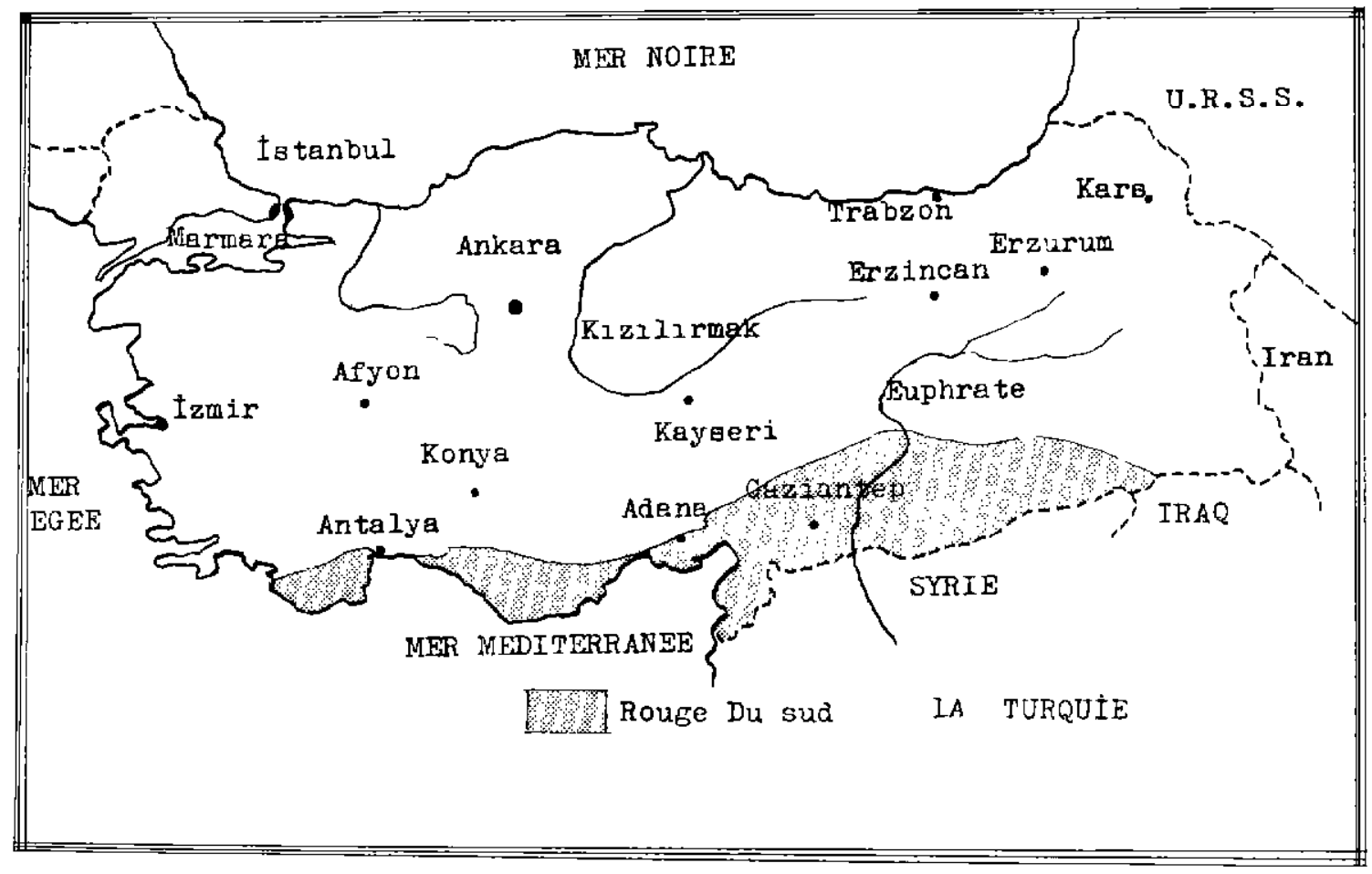

Fig. 4. 
Mardın et Surt. Ces régions séparées de la mer । par les monts Anti-Taurus annoncent, vers le Sud, le désert syrien. Les pluies n'y sont pas plus abondantes que sur le plateau anatalien, mais la température y est beaucoup plus douce en hiver et beaucoup plus torride en été. En 1960, II n'est tombé que $189 \mathrm{~mm}$ d'eau dans le pluviomètre de la ferme de CEYLAN-PINAR, au sud d'Urfa. ta saison sèche peut durer de 4 à 5 mols.

C'est dans ce milieu que la race Rouge du Sud montre ses qualités. Parfaltement adaptée à cette chaleur et à cette sécheresse, elle contınue à donner des rendements en lait élevés sı elle est correctement alimentée.

Cette résistance exceptionnelle à la chaleur confirme l'idée d'un ancêtre zébu dans les orlgines de la race : sa conformation spécrale, sa cuisse plate et démesurément longue, sa bosse rudimentaire, confirmeraient cette filiation.

Les quelques mensurations d'animaux de cette race, prises sur 100 vaches au dépôt de bovins de Boztepe (près d'Antalya) par SERAFETTIN YAVUZ entre 1948 et 1956 confirment les données du Tableau I.

Une étude a été falte au haras de Cukurova par CEMAL NADI AYTUG sur les productions latières de cette race. Ont été calculés : la production laıtıère annuelle, la longueur de la période de lactation, le taux moyen butyreux, la production selon les mols de l'année et l'effet de l'âge sur la lactation. On a étudié aussı la corrélation entre la production laıtıère de la première lactation et l'âge de production maximum.

Le matériel d'étude a porté sur 215 vaches de 52 familles. La production moyenne annuelle, lo durée de la lactation et les variations de production selon les mols de lactation ont été calculées, en 1948, sur 41 vaches. Les facteurs de conversıons, corrélatıons et régressıons ont été étudiés sur les lactations de 42 vaches de la 4 e année à la $7 \mathrm{e}$ année. Ces vaches avalent été sélectıonnées parmi les 216 du troupeau.

La moyenne de production annuelle des melileures vaches est de $2.416 \pm 84,2$ litres. Lo moyenne de production corrigée selon les âges est $2.670,73 \pm 87,885$. La période moyenne de produclion est de 274,4 jours $\pm 4,07$ et la moyenne de matière grasse est de $3,515 \pm 0,02$ p. 100 .

La production aux $3 \mathrm{e}, 4 \mathrm{e}, 5 \mathrm{e}$ mois de lactation est plus élevée que la moyenne, mass la plus importante se situe au 3 e mois.
La production de lait de la $4 \mathrm{e}$ à la $7 \mathrm{e}$ lactation (entre 5 et 9 ans) est plus élevée que les autres: elle est maximum à la 4 e.

La production décroît après la 7 e lactation.

En 1967 au Dépôt de Bovins de Boztepe (Antalya) l'élevage de ces deux races a donné les résultats sulvants :

La race Rouge du Sud:

Nombre de vaches sallies......... 119

Nombre de nouveau-nés ........ 96

80,6 p 100 de nassance

Mortalıté des nouveau-nés......... 3,1 p. 100

Lo race hollandaise :

Nombre de vaches saillies.......... 84

Nombre de nouveau-nés... ...... 75

89,2 p. 100 de naıssance

Mortalıté des nouveau-nés ........ 8 p. 100

A Boztepe la production laitière annuelle moyenne chez la Rouge du Sud a été de 1.800 $2.000 \mathrm{kgs}$ de laıt, en 1967.

La production annuelle chez la Hollandaise a atteınt $3.000 \mathrm{~kg}$ mass les vaches hollandasses reçoivent $5 \mathrm{~kg}$ de concentré par jour sous forme de son, d'orge et de tourteau, alors que les Rouges du Sud dorvent se contenter de manger les pâturages de la station. On peut estimer que ce concentré supplémentaıre représente la valeur d'une production laıtière de 12 à 15 kılos de laıt, ce qui, pour une lactation de 200 jours seulement, équivaudraıt à un total de 2.500 à 3.000 kilos de lait. On est lorn du compte.

Dans ce Dépôt de bovins, dans des conditions assez médiocres d'entretien, en 1961-1962, la production moyenne atteignait $2.100 \mathrm{~kg}$ pour 200 vaches laitıères Rouge du Sud. SI I'on compare sur le pian économique, l'avantage revient sans conteste à la race Rouge du Sud.

Le principal reproche que l'on pourrait sans doute adresser à cette race serait la faible teneur de son lait en matière grosse, ce qu'on ne lui reproche pas en Syrie et au Liban. A la ferme de CEYLAN-PINAR, où I'on élève 160 vaches laltières, on a pourtant obtenu $4.000 \mathrm{~kg} \mathrm{de}$ moyenne avec un toux butyreux de 3,6 p. 100. Une vache championne de cette même ferme a fourni $5000 \mathrm{~kg}$ de lait en une lactation.

If convient également de souligner la résıstance de la race à la chaleur. Des comparalsons ont pu être effectuées entre la race Rouge et la 
race Frisonne. Nous donnons ici les éléments relevés le 23 septembre 1968 à 9 h 30 au dépôt de Boztepe situé près d'Antalya. II s'agıt des températures rectales ef du nombre de mouvements respıratoires relevés sur des anımaux à l'étable.

\begin{tabular}{|c|c|c|}
\hline & $\begin{array}{l}\text { Température } \\
\text { rectale } \\
\text { - }\end{array}$ & $\begin{array}{l}\text { Mouvements } \\
\text { respiratoires } \\
\text { par mınute }\end{array}$ \\
\hline $\begin{array}{l}\text { Trols troupeaux Hollandals } \\
\text { nés sur place en } 1965 \text { et } \\
1967 \text { et un troupeau Im- } \\
\text { porté en } 1964 \ldots \ldots \ldots \text {. }\end{array}$ & $\begin{array}{c}39,2 \\
(38,8-40)\end{array}$ & $\begin{array}{c}38 \\
(29-48)\end{array}$ \\
\hline $\begin{array}{l}\text { Trois taureaux de race } \\
\text { Rouge nés sur place en } \\
1962-1965 \text { et } 1967 \ldots \ldots \text {. }\end{array}$ & $\begin{array}{c}38,8 \\
(38,5-39,5)\end{array}$ & $\begin{array}{c}25 \\
(21-28)\end{array}$ \\
\hline
\end{tabular}

Le même jour on a pu faire d̀ 10 h 30 les observations suivantes sur des femelles au pâturage, en plein soleil.

\begin{tabular}{|c|c|c|}
\hline & $\begin{array}{l}\text { Température } \\
\text { rectale } \\
-\end{array}$ & $\begin{array}{l}\text { Mouvements } \\
\text { respiratoires } \\
\text { par minute }\end{array}$ \\
\hline $\begin{array}{l}\text { Six vaches Hollandaises } \\
\text { nées à Boztepe en } 1965 \text { et } \\
\text { Cinq vaches importées en } \\
1961-1962 \text { et } 1963 \ldots \ldots \text {. }\end{array}$ & $\begin{array}{c}39,4 \\
(39-40,6)\end{array}$ & $\begin{array}{c}52 \\
(32-72)\end{array}$ \\
\hline $\begin{array}{l}\text { Six vaches de race Rouge } \\
\text { nées d̀ Boztepe en 1957- } \\
1960 \text { et } 1968 \ldots \ldots \ldots \ldots\end{array}$ & $\begin{array}{c}38,9 \\
(38,7-39,1)\end{array}$ & $\begin{array}{c}39 \\
(28-52)\end{array}$ \\
\hline
\end{tabular}

Dans la régron d'Antalya, en juillet et en coôt, les animaux de la race hollandase respirent la bouche ouverte et la langue pendante un peu comme des chiens.

Parm les vaches hollandaises observées au pâturage, une seule avait une respiration à peu près normale (32 mouvements/mn). Toutes les autres présentaient un rythme extrêmement précipité. Elles semblaient inquiètes, recherchaient l'ombre et souffraient visiblement de l'ardeur du soleil. Cecı s'observe d'alleurs déjà dès le mols de mal. L'un de nous en avalt été frappé. Au contraire, les vaches de la race Rouge du Sud Anatolie, bien qu'ayant un rythme respiratoire plus élevé que la normale, demeurent calmes et restent au pâturage en pleın solell, jusqu'aux environs de midi.

On peut donc, grâce à ces simples observations comme en comparant les performances des deux races, se rendre compte des avantages de la race Rouge. Pourtant, cette race a malheureusement diminué très sérieusement en nombre. Au recensement de 1960, on en comptait 676.000. En 1962, il n'y en avalt plus que 538.000 soit 138.000 de mons, un peu plus de 20 p. 100 . Lo. race perd aussi en qualité car on ne la sélectionne absolument pas.

Les risques de régression et même de disparition de cette belle race en Turquie sont déjà très apparents. Dans les régions bordant la Syrie, il existe encore une forme de proprlété anachronique avec les «Agas» (Seigneurs) qui possèdent d'ımmenses étendues de terre englobant souvent plusieurs villages. Jusqu'à ces dernières années, l'Aga avalt besoln du paysan et de ses animaux pour cultiver les champs et utiliser les pâturages. II s'étalt formé une sorte d'associatıon, genre de mélayage entre les paysans et le sergneur, celui-cl prêtant ses champs et donnant les semences, celui-là apportant ses bras et ses animaux de traval. Les gains de la récolte étalent divisés en deux parties égales et le paysan y a outait le revenu de ses vaches et de ses moutons.

Deux facteurs sont récemment intervenus qui modifient ces coutumes de fond en comble. Le premier est le prix du blé, porté à 80 kurus le $\mathrm{kg}$, solt sensiblement le prix européen, alors que le prix du bœuf sur pled est $280 \mathrm{TL} *$ les $100 \mathrm{~kg}(1)$. Ce déséquilibre a poussé l'« Ağa» à labourer toutes ses terres, même si elles sont manifestement impropres à la culture, même si les récoltes, très aléatorres, dépendent uniquement de la pluie.

— «S'il pleut, disent les paysans, on récolte 10 à 15 fars la semence, mais sı l'année est sèche, c'est seulement 3 à 4 fols que la terre rapportera.»

Autrement dit, en bonne année, on obtiendra 10 à 15 quintaux à l'ha i en mauvaise année, 3 à 4 quintaux.

Le deuxième facteur qui favorise l'homme puissant est la mécanisation agricole. Elle l'a rendu indépendant du paysan du village. Aınsi, grâce à ces immenses cultures, l'Ağa n'a plus le soucl de survellance de ses troupeaux. On laboure et on sème avec les tracteurs, on récolte d̀ la molssonneuse-batteuse sans aucune sujétıon à l'égard des habitants des villages qui

(1) France : $308-310 \mathrm{~F}$ soit $620 \mathrm{TL}$ environ.

* Livre Turque. 
n'ont plus d'autres ressources que de partir et d'abandonner leurs élevages. Sans souci également de la conservation du sol, ce bien précieux. C'est pourquoi la race Rouge du Sud est partout en régression.

Les enquêtes conduites par FIASSON et Ferıdum GORGULU dans les villages des provinces d'Urfa et de Mardin ont montré que, par suite des mauvaises conditions d'alımentation et d'entretıen, les vaches ne donnent guère qu'un veau tous les deux ans et même pas toujours. Les taureaux sont trop jeunes, famélıques ou absents. Le Ministère de l'Agriculture falt des distributions gratuites de taureaux dans quelques villages, mais les paysans trop pauvres se refusent à les nourrir et ces animaux se trouvent rapidement dans un état lamentable les rendant impropres à la reproduction. D'ailleurs, les vaches elles-mêmes sont si mal nourries qu'elles sont incapables de gestations régulièrement et normalement espacées tous les quatorze mois Elles ont besoin de longs repos après le part tout comme les terres ont besoin d'être laissées un an en jachère avant de pouvar porter une autre récolte.

A plusieurs reprises l'attention des responsables du service de l'élevage au Minıstère turc de l'Agriculture a été attirée sur les qualités de cette race si bien adaptée à son milieu et susceptıble par la sélection de remarquables performances qui n'ont pas encore été totalement extérıorısées. On a en effet envisagé d'abandonner la sélection de cette race dans les établissements officiels et de l'absorber par crolsement, solt avec la Frisonne au Dépôt de Bovins de Boztepe, près d'Antalya, soit par crossement avec la race de Guernesey au haras de Cukurova, près d'Adana. Agır aınsı serait, à notre avis, une très grave erreur.

\section{CONCLUSIONS}

Une fors de plus, II apparaît qu'on a tendance d̀ sacrifier la réalité aux mythes. Nı l'état des praıries, ni l'évolution de l'élevage, nı la technıcité des éleveurs ne permettent l'entretien de races très perfectionnées et très exıgeantes dans la régıon Sud de l'Anatolie. Alors qu'un effort coordonné et survi pourrart, non seulement permettre d'améliorer une race intéressante, mais encore d'obtenir des sujets aptes à l'exporta- tıon, par là même d'enrichir la région et, au-deíà, de constituer une ressource en devises, on s'obstine à importer une race étrangère, inadaptée et inadaptable sans investissements exagérés.

On retrouve icl les erreurs commises dans d'autres pays. D'une part, beaucoup de gens estıment qu'ımporter des races déjà très évoluées permet d'éviter d'accomplır un long et coûteux travail de sélection. D'autre part, ceux qui vantent la qualité des races perfectionnées de leur propre pays ḋ l'étranger font le plus souvent abstraction de l'importance du nouveau milieu dans lequel la race va se trouver placée. Il n'y a pas que la sélection qui comple. Il y a aussi l'alımentatıon, I'hygiène générale. les aptitudes des éleveurs. On ne parle jamals de cela. Ce n'est pas parce que la race bovine Frısonne qu'elle soit de provenance française, canadienne, américanne et, bren entendu, hollandaise, est une excellente race, qu'elle doit réussir partout même dans des régions chaudes aux ressources alimentaires limitées. Qu'on se souvienne de l'effort accompli sur les plans de la génétique, de l'alımentation, de l'hygiène générale pour obtenir et pour conserver les qualités de cette race ou d'autres races analogues. Les races ne peuvent prétendre à l'universalité qu'autant qu'elles vivent dans un milieu à peu près semblable à celui où elles se formèrent et qu'elles sont exploitées par des hommes pratıquant des techniques analogues à celles des éleveurs de leur bercecu de race.

Il existe à la base de la zootechnie une série de règles et d'axiomes sımples. Nous en citerons ou en rappellerons quelques-uns que tout directeur d'élevage devrait avorr sans cesse présents à l'esprits.

10 Productivité n'est pas fatalement rentabilité.

$2^{\circ}$ La race, c'est le produit de l'hérédité par le bınôme milıu - alımentatıon, mis en œuvre par des éleveurs avertıs.

$3^{\circ}$ On ne peut sélectionner si l'on ne sait pas et sil'on ne peut pos alimenter.

Enfin cecl emprunté d̀ P. DECHAMBRE, qui résume un peu tout : « Les anımaux les meilleurs ne sont pas nécessairement les plus perfectıonnés mais ceux qui sont le mieux adaptés aux différentes conditions du mode d'exploitation qu'ils subissent. 》 


\section{SUMMARY}

Tha red cattla breed of South Anatolia (Güney Anadolu Kirmizisı)

The authors describe the breed, from Syrian and Lebanon, where it is named: Beledie breed of Damas or Alep. Perfectly adapted to the heat and to the dryness, it contınues to give high milk yields if it is well fed. The annual milk production (2416 $\doteq 84.2)$ the lactation period $(274.4 \pm 407 \mathrm{j}$.), the medium buttery rate ( $3.515 \pm 0.02 \mathrm{p} .100$ ), the production according to the months of the year and the age effect on the lactation are calculated from 215 cows of 52 families

Therefore, the authors consider that the improvment of an interesting breed and the production of suitable animals for exportation should be possible by a joint and coherent effort of selection and they expose that it is an error to import advanced breeds to avold a long work of selectıon, sacrificing the reality to the myth.

\section{RESUMEN}

La raza bovina roja del sur de la Anatolıa (Gủney Anadolu Kirmizısi)

Los autores describen la raza, originaria de Siria y de Libano donde se la denomına : raza Bélédie de Damas o de Alep. Perfectamente adaptada al calor y a la sequedad, sigue dando rendimientos elevados de leche con tal de que se la alimente bien. Se calculan la producción lechera anual $(2416 \pm 84,21$.), la duración de lactación $(274,4 \pm 4,07 \mathrm{~d}$ ), el termıno medio butiroso $(3,515 \pm 0,02 \mathrm{p} 100)$, la producción según los meses del año y el efecto de la edad sobre la lactación en 215 vacas perteneciendo a 52 famılias.

En sus conclusiones, los autores indican que un esfuerzo de selección coordenado y contınuo permıtıria mejorar una raza interesante y obtener anımales aptos para la exportación y mostran la equrvocación según la cual se importan razas muy evolucionadas para evitar un largo trabajo de selección, sacrificando ası la realidad al mito.

\section{BIBLIOGRAPHIE}

EDDI (J.). - Géographie de la Syrie et du Liban. Beyrouth 1939.

FERRANDO (R.). - Le Lait, $1941 ; 21$ (209-210); 346-361.
SABA (M.). - L'Inspection des viandes et des abattoirs en Syrie. Thèse Dr. Véter., Paris, 1936. 